\title{
Inserção do profissional farmacêutico na atenção básica de saúde: uma revisão
}

\section{integrativa}

\author{
Insertion of the pharmaceutical professional in basic health care: an integrative review \\ Inserción del profesional farmacéutico en la atención básica de salud: una revisión integrativa
}

Recebido: 19/09/2021 | Revisado: 29/09/2021 | Aceito: 05/10/2021 | Publicado: 09/10/2021

\author{
Jhennyffer de Sousa Lima Costa \\ ORCID: https://orcid.org/0000-0002-7532-9869 \\ Faculdade Integrada Carajás, Brasil \\ E-mail: jhennyffer1988@gmail.com \\ Yolanda de Jesus Morais \\ ORCID: https://orcid.org/0000-0001-7105-8267 \\ Universidade Federal do Pará, Brasil \\ E-mail: yolandamorais123@gmail.com
}

\begin{abstract}
Resumo
Objetivo: bibliograficamente, objetiva verificar a inserção do profissional farmacêutico na atenção básica de saúde demonstrando seu papel. Métodos: de natureza bibliográfica, mediada a partir de estudos primários recrutados de artigos e periódicos de cunho científico, preconiza o método de revisão integrativa descritiva, com abordagem qualiquantitativa. Resultados: mediante a análise de dados aqui recrutados foi perceptível determinar que a inserção do profissional farmacêutico na atenção básica de saúde é de fundamental importância para a saúde pública da federação brasileira. Conclusão: A evidências que ações, voltadas para a inserção do profissional do profissional farmacêutico é de suma relevância, ora que, corrobora para uma melhor qualidade de vida dos indivíduos requerentes de atendimentos da atenção primária de saúde. Outro fator preponderante é que a inserção farmacêutica tem influenciado muito sobre a atenção básica de saúde, fazendo-se necessário que as gestões de saúde pública prezem os serviços prestados por estes colaboradores da saúde humana, ora que assim minimize-se os usos incorretos de medicamentos prevenindo possíveis danos à saúde pública.
\end{abstract}

Palavras-chave: Atenção farmacêutica; Atenção Primária; Atenção Básica; Medicamentos.

\begin{abstract}
Objective: bibliographically, it aims to verify the insertion of the pharmacist in primary health care, demonstrating their role. Methods: bibliographical in nature, mediated from primary studies recruited from scientific articles and journals, it advocates the descriptive integrative review method, with a quali-quantitative approach. Results: through the analysis of data recruited here, it was noticeable to determine that the insertion of the pharmacist in primary health care is of fundamental importance for the public health of the Brazilian federation. Conclusion: The evidence that actions, aimed at the insertion of the professional pharmacist, is of paramount relevance, which corroborates for a better quality of life of individuals requesting care in primary health care. Another important factor is that pharmaceutical insertion has greatly influenced primary health care, making it necessary for public health management to value the services provided by these collaborators of human health, so that the misuse of medicines is minimized preventing possible harm to public health.
\end{abstract}

Keywords: Pharmaceutical care; Primary Attention; Basic Attention; Medicines.

\section{Resumen}

Objetivo: bibliográficamente, se busca verificar la inserción del farmacéutico en la atención primaria de salud, demostrando su rol. Métodos: de carácter bibliográfico, mediado a partir de estudios primarios reclutados a partir de artículos y revistas científicas, aboga por el método de revisión descriptiva integradora, con enfoque cuali-cuantitativo. Resultados: a través del análisis de los datos aquí recogidos, se notó determinar que la inserción del farmacéutico en la atención primaria de salud es de fundamental importancia para la salud pública de la federación brasileña. Conclusión: La evidencia de que las acciones, encaminadas a la inserción del profesional farmacéutico, son de suma relevancia, lo que corrobora para una mejor calidad de vida de los individuos que solicitan atención en la atención primaria de salud. Otro factor importante es que la inserción farmacéutica ha influido mucho en la atención primaria de salud, haciendo necesario que la gestión de la salud pública valore los servicios prestados por estos colaboradores de la salud humana, de manera que se minimice el mal uso de los medicamentos previniendo posibles daños a la salud pública.

Palabras clave: Atención farmacéutica; Atención Primaria; Atención Básica; Medicamentos. 


\section{Introdução}

A inserção do profissional farmacêutico na atenção básica de saúde tem sido evidenciada como uma competência de cunho teórico que conduz a uma prática clínica-eficaz, ora que, ocorre uma coordenação da terapêutica medicamentosa de maneira mais precisa e segura para o paciente. As responsabilidades farmacoterapêuticas devem ser assumidas por esses colaboradores da saúde humana/, sendo empregados valores e atitudes que venham disponibilizar um tratamento para o paciente de forma globalizada e integral (Vieira, 2010).

Quando considerado o papel primordial dos profissionais supramencionados, cabe destacar que, os mesmos realizam a identificação, prevenção e resolve problemas alusivos ao uso de medicamentos. Sendo possível verificar se todos os medicamentos que os pacientes estão fazendo uso, são de fato seguros e efetivos, fomentando assim a respectiva adesão ao tratamento oferecido. Posteriormente, o farmacêutico delibera se o paciente explicita algum problema relacionado ao uso de medicamentos, cria planejamento de cuidados que venham soluciona-lo, caso necessário, em parceria com a equipe multiprofissional de saúde e, subsequentemente, verifica os resultados dessa intervenção (Maciel, 2018).

Fomenta-se que, a introdução farmacêutica foi aderida no Sistema Único de Saúde (SUS) através da Política Nacional de Medicamentos e devido a Política Nacional de Assistência Farmacêutica e suas respectivas complementares. Para Neto e Borges (2020), ao realizarem um estudo por meio da análise de dados textuais puderam determinar que, as políticas públicas de saúde do país requerem participação ativa dos profissionais, ora citados, visando proteção, recuperação e promoção da saúde, bem como possível prevenção de agravos.

Silva e Torres (2019), destacam que o farmacêutico possui papel fundamental na atenção básica de saúde, visto que, o mesmo proporciona um trabalho que visa atuar na prevenção de agravos de indivíduos que realizam uso irracional de medicamentos. Com isso, percebe-se que a atuação dessa área laborativa busca oferecer um serviço mais seguro visando a qualidade de vida dos pacientes e/ou usuários do SUS, bem como oferece confiabilidade na utilização dos fármacos.

Dessa forma, vale frisar que a presente revisão integrativa apresenta relevância, pois demonstra como ocorre a inserção do farmacêutico na atenção básica, bem como seu respectivo papel. Oferecendo assim informes cruciais para a execução de um serviço de qualidade que tenha como principal objetivo instruir os indivíduos para o uso racional dos medicamentos. Outro fator primordial é que a execução desse estudo viabiliza vastas informações que auxiliam na execução das atividades dos profissionais aqui citados, bem como fornece informes para as autoridades públicas que podem com isso implementar ações que venham contribuir nessa atuação para que seja cada vez mais integral e, individualizada que busque fornecer prevenção no uso irracional dos medicamentos.

Considerando à vasta gama de pesquisas publicadas alusivas ao tema aqui apresentado os pesquisadores ao verificarem que existe uma certa afinidade pelo tema proposto iniciaram o presente projeto. Outro quesito significativo foi que atendandose a atenção básica de saúde da federação brasileira, bem como vendo a importância de agregar dados sobre a inserção dos farmacêuticos e suas contribuições na saúde pública os pesquisadores buscaram oferecer informações resumidamente de diversos trabalhos com temas análogos favorecendo assim na construção de uma possível revisão integrativa.

Outro fator relevante é que sabe-se que ainda existe um aumento considerável de indivíduos realizando uso irracional de medicamentos e na acessibilidade do profissional farmacêuticos na atenção primária pode mudar significativamente esse cenário. Visto que, sabe-se que o uso incorreto de medicamentos pode ser tornar um sério problema de saúde pública. Portanto, um dos objetivos desses profissionais da saúde citados acima é alertar sobre os riscos do uso incorreto e intervir sobre tal conduta alertando a sua gravidade e tentando adequar o plano terapêutico ofertado para um certo indivíduo.

Ressalta-se que o bem-estar biopsicossocial das pessoas é um fator crucial para a saúde pública em geral, assim determina-se a atuação multidisciplinar dessa área que visem ações voltadas ao sistema de saúde pública do Brasil. Diante o 
exposto, surgiu a pergunta norteadora do presente estudo: Qual a importância da atuação do profissional farmacêutico na atenção básica de saúde?

A pesquisa aqui empregada foi norteada a partir do recrutamento de dados procedentes do Google acadêmico, bem como da Biblioteca Virtual de Saúde (BVS). O questionamento de base que surgiu para sintetizar o conteúdo dos dados recrutados fora: Qual a importância da atuação do profissional farmacêutico na atenção básica de saúde? Diante o exposto verifica-se que a construção o presente projeto está voltado para a resolução da problemática caracterizada anteriormente.

\section{Metodologia}

\subsection{Tipo de estudo}

A presente pesquisa foi desenvolvida a partir de uma revisão integrativa da literatura, com abordagem de natureza descritiva e quantitativa. Sabe-se que a revisão integrativa possui etapas norteadoras para sua execução e, considerando esse fator, surgiu a primeira etapa que foi a elaboração da pergunta: Qual a importância da atuação do profissional farmacêutico na atenção básica de saúde?

Souza, Silva e carvalho (2010), afirmam que a revisão integrativa tem sido classificada como um mecanismo ímpar na área da saúde, ora que sintetiza os trabalhos disponíveis sobre uma determinada temática e orienta a prática baseando-se em conhecimento científico. A análise de dados, detém diminuição de vieses e erros, explicitando que a revisão integrativa é um instrumento válido da prática que é baseado em evidências.

\subsection{Coleta de dados}

A segunda etapa ocorreu mediante a busca de amostras na literatura, fator esse, intrinsecamente relacionado a fase mencionada acima. As bases de dados de cunho eletrônico, exploradas fora: Google acadêmico e BVS o que proporcionou uma busca ampla e múltipla. A partir da exploração da base de dados da BVS fez-se possível obter artigos da Medical Literature Analysis and Retrieval System Online (MEDLINE), Scientific Electronic Library Online (SCIELO), PubMed e Literatura Latinoamericana e do Caribe em Ciências da Saúde (LILACS) e do periódico Ensaio e Ciência. Salienta-se que a determinação dos critérios de inclusão do presente estudo esteve sempre inerentemente de acordo com a pergunta norteadora, considerando-se assim os dados, participantes, intervenções e resultados das pesquisas consideradas.

Cabe mencionar que os dados elaborados para o presente escrito foram subsidiados mediante a leitura minuciosa de publicações teórica-científicas viabilizadas no período de 2015 a 2020, fator determinante da frequência dos trabalhos alusivos à temática aqui levantada. Todavia, a determinação ocorreu mediante observação do título, do ano de publicação, idioma, natureza, base de dados, amostras, tipo de estudo, região de estudo e coleta das amostras. Além desses fatores anteriormente citados, fora verificado as etapas das pesquisas, sendo analisada detalhadamente o resumo, introdução, metodologia, resultados, discussão, conclusão e referências, tornando-se propício um estudo singular de cada pesquisa aqui explorada, o que determinou a terceira fase, conforme Tabela 1. 
Tabela 1 - Caracterização das etapas analisados nos artigos aqui considerados.

\begin{tabular}{|l|l|}
\hline 1) Título & $09)$ Região de estudo. \\
\hline 2) Ano de publicação & $10)$ Resumo \\
\hline 3) Idioma & $11)$ Introdução \\
\hline 4) Natureza & 12) Metodologia \\
\hline 5) Base de dados & 13 ) Resultados \\
\hline 6) Amostras & 14) Discussão \\
\hline 7) Tipo de estudo & $15)$ Considerações finais \\
\hline 8) Coleta das amostras & $16)$ Referências \\
\hline & \\
\hline
\end{tabular}

Fonte: Inserção do profissional farmacêutico na atenção básica de saúde: uma Revisão Integrativa,( 2021).

\subsection{Critérios de inclusão e exclusão}

Ainda sobre a terceira fase que fora empregada, acentua-se que a extração dos artigos aqui designados, faz-se necessário, posto que utilizou-se como instrumento os anais citados, verificando a integralidade dos artigos que detiverem informes de maneira integra e completa, garantindo assim precisão na análise das informações que pretende-se extrair. Com isso, determinouse os seguintes critérios de seleção: artigos científicos que apresentaram informes a título original, que estivessem publicados no idioma português e/ou inglês no período de 2015 a 2020, contribuindo assim para a conclusão da quarta etapa que foi obedecida na presente revisão. Destaca-se que o recrutamento dos dados aconteceu no ano de 2021, mais precisamente no início do mês de maio. Os artigos com temas antagônicos dos aqui propostos e publicados fora do período descrito acima foram excluídos da revisão integrativa.

Reforça-se que para a reunião dos artigos científicos aqui mensurados, utilizou-se os descritores que estão adequadamente presentes em Ciências da Saúde (DeCS), a título de exemplo: acessibilidade farmacêutica, atenção básica de saúde, farmácia e uso irracional de medicamentos. Como foi explorado as bases da BVS, foi empregados os operadores Booleanos, a título de exemplo: AND e $O R$.

\subsection{Análise de dados}

A análise crítica dos artigos científicos aqui alistados constituiu a quarta fase da presente revisão, onde demandou-se uma abordagem de caráter organizado, ponderando assim rigorosamente as peculiaridades de cada pesquisa considerada. Mensura-se que os métodos, bem com os resultados foram bem averiguados comparando quais são suas respectivas utilidades na prática profissional dos farmacêuticos na atenção básica de saúde humana.

Destaca-se que a análise dos dados, bem como a organização dos mesmos foi realizada mediante uso de tabelas, onde o programa Microsoft Excel ${ }^{\circledR}$ e, por se tratar de uma pesquisa levantada mediante dados públicos e disponibilizados gratuitamente de maneira remota, cabe reforçar que a mesma não necessitou de aprovação em Comitês de Ética em pesquisa.

\section{Resultados}

Faz-se perceptível que mediante a análise de dados aqui recrutados a inserção do profissional farmacêutico na atenção básica de saúde é de fundamental importância para a saúde pública da federação brasileira. Cabe ainda fomentar que 
primeiramente foram recrutados 35 trabalhos, todavia após análise detalhada dos mesmos apenas 12 trabalhos explicitavam temáticas capazes de resolver a situação problema, ora aqui incialmente levantada, conforme expresso na Figura 1.

Figura 1 - apresentação esquemática dos critérios seletivos.

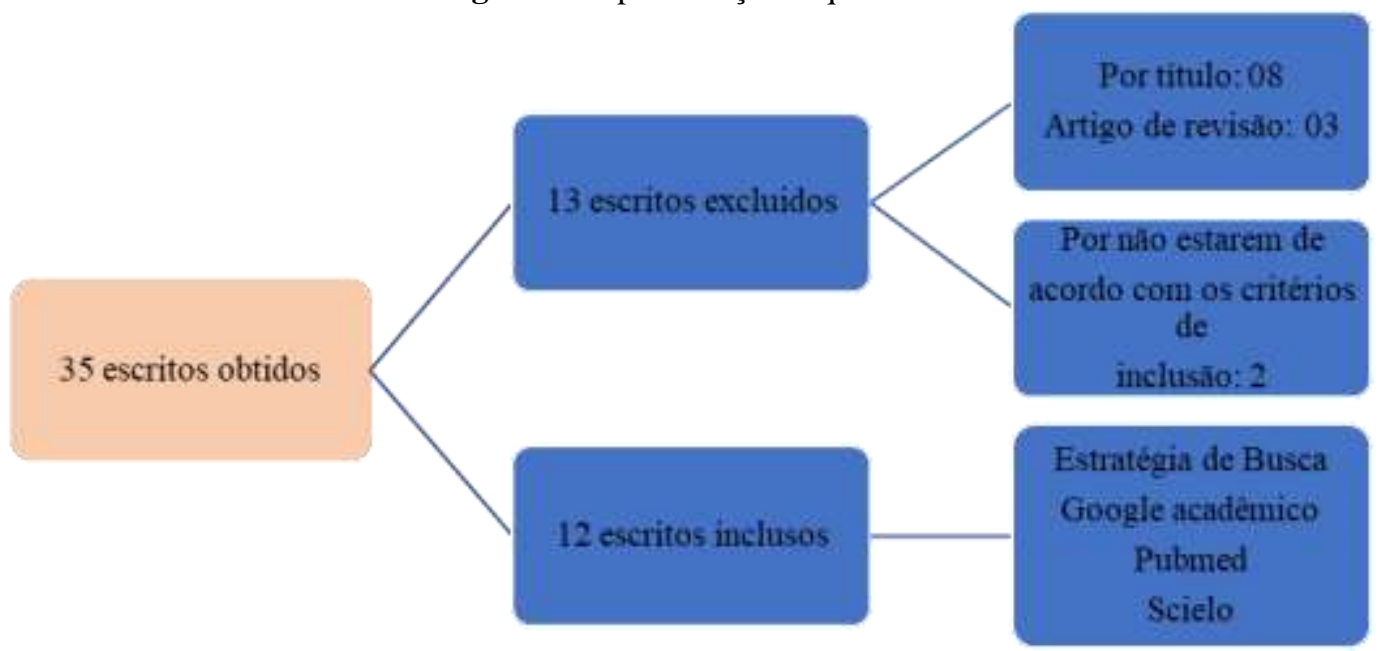

Fonte: Inserção do profissional farmacêutico na atenção básica de saúde: uma Revisão Integrativa, (2021).

Para a consecução da presente revisão integrativa considerou-se estudos publicados no período de 2017 a 2020 , sendo que, o maior índice de publicação foi no ano de 2018, detendo 33,33\% (n=4) dos achados. Considerando a variável do ano proposto no presente estudo cabe frisar que se apresentou um incremento apenas ano de 2018, conforme supramencionado, e de modo geral as publicações foram realizadas de forma oscilatória Gráfico 1. Ressalta-se ainda que foi possível alistar as seguintes informações: 91,66\% (n=11) das amostras, trata-se de artigos científicos e 8,33\% $(n=1)$ uma tese de doutorado.

Gráfico 1 - Apresentação dos números de trabalhos publicados no período proposto.

\section{Número de trabalhos publicados}

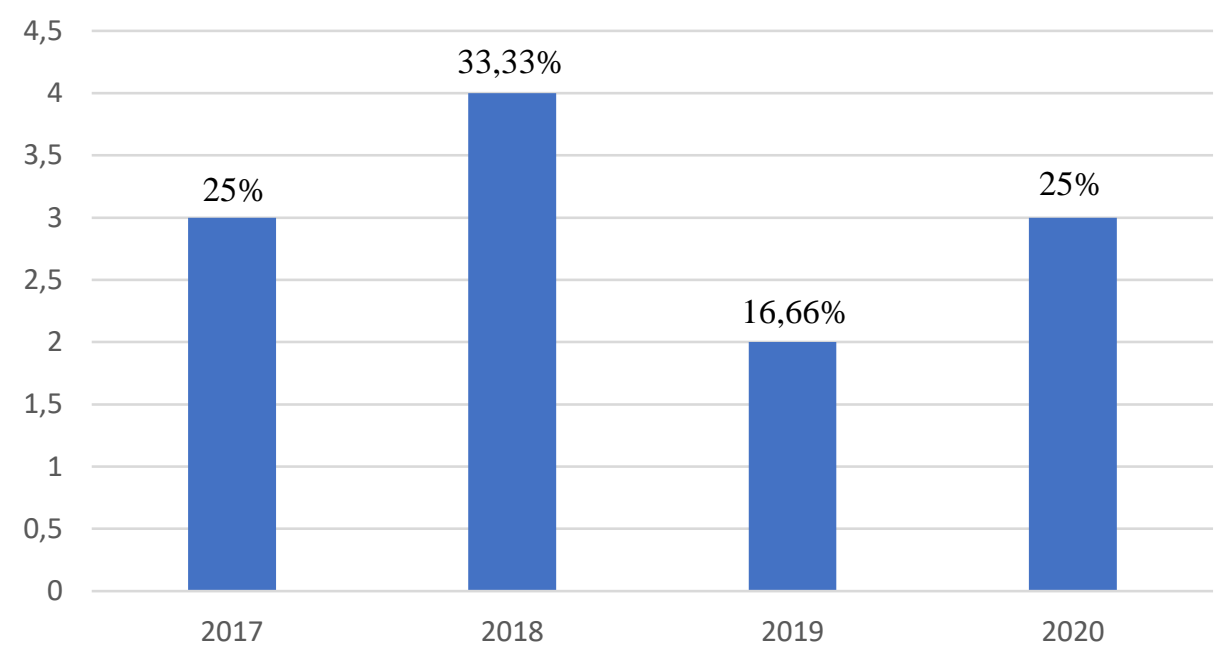

Fonte: Inserção do profissional farmacêutico na atenção básica de saúde: uma Revisão Integrativa, (2021).

Outro fator preponderante, ora aqui, averiguado fora as bases de dados utilizadas. Fomenta-se que das pesquisas aqui minuciosamente analisadas 33,33\% (n=4) foram advindas do Google Acadêmico, 8,33\% (n=1) LILACS, 58,33\% (n=7) Scielo. Entretanto, diante dos informes mensurados foi possível determinar a relevância da inserção do profissional farmacêutico no 
âmbito da saúde pública primária. Os artigos analisados explicitam os manejos realizados por estes profissionais aqui considerados, sendo evidente a importância da prática farmacêutica na saúde primária.

Vale destacar que dentre os 12 trabalhos averiguados, 41,66 \% (n=5) explicitavam assuntos concernentes aos serviços, atenção e práticas farmacêuticas, $8,33 \%(\mathrm{n}=1)$ força de trabalho, 16,66 $(\mathrm{n}=2)$ tratou-se de assuntos condizentes ao avanço e desafio, $8,33 \%(n=1)$ a promoção do acesso e ao uso irracional de medicamentos e $16,66 \%(n=2)$ relacionados ao cuidado e assistência, destaca-se que todos mencionados anteriormente apresentavam conceitos de assistência farmacêutica, estando inclusive devidamente mencionada nos títulos conforme Tabela 2.

Em contrapartida cabe mencionar que um dos trabalhos recrutado e analisado não apresentava no seu título as palavras assistência farmacêutica, todavia o mesmo fez-se necessário para construção da presente revisão uma vez que a mesma traz conceitos do SUS, e o artigo apresentava informes concernentes a descentralização e regionalização da política de saúde sendo mencionado a relevância da inserção farmacêutica no âmbito da saúde primária.

Tabela 2 - Distribuição segundo Autores, ano e títulos dos trabalhos originais recrutados.

\begin{tabular}{|c|c|c|}
\hline Autoria & Ano & Título \\
\hline Caetano, Silv & 2020 & $\begin{array}{l}\text { Serviços farmacêuticos na atenção primária em saúde à luz do modelo ambiguidade - } \\
\text { conflito. }\end{array}$ \\
\hline $\begin{array}{l}\text { Molina, Hoffmann \& } \\
\text { Finkeler }\end{array}$ & 2020 & Ética e assistência farmacêutica na atenção básica: desafios cotidianos. \\
\hline Neto \& Borges & 2020 & A prática da integralidade no cuidado farmacêutico na atenção primária à saúde. \\
\hline Carvalho \& Leite & 2017 & Força de trabalho na assistência farmacêutica da atenção básica do SUS, Brasil. \\
\hline Costa et al. & 2017 & Avanços e desafios da assistência farmacêutica na atenção primária no SUS. \\
\hline Melo \& Castro & 2017 & $\begin{array}{l}\text { A contribuição do farmacêutico para a promoção do acesso e uso racional de medicamentos } \\
\text { essenciais no SUS. }\end{array}$ \\
\hline Lopes, Staub \& Ross & 2018 & $\begin{array}{l}\text { Atenção farmacêutica e a humanização da assistência: a influência do profissional } \\
\text { farmacêutico na acessibilidade à informação. }\end{array}$ \\
\hline Maciel & 2018 & Atuação cotidiana do farmacêutico na atenção primária à saúde. \\
\hline Pereira et al. & 2018 & $\begin{array}{l}\text { Descentralização e regionalização da política de saúde abordagem histórico-comparada } \\
\text { entre o Brasil e Espanha. }\end{array}$ \\
\hline $\begin{array}{l}\text { Silva Mendonça, } \\
\text { Oliveira \& Chemello. }\end{array}$ & 2018 & À prática clínica do farmacêutico no núcleo de apoio a saúde a família. \\
\hline Barros & 2019 & Serviços farmacêuticos clínicos na atenção primária à saúde do Brasil. \\
\hline $\begin{array}{l}\text { Barberato, Scherer \& } \\
\text { Lacourt }\end{array}$ & 2019 & O farmacêutico na atenção primária no Brasil: uma inserção em construção. \\
\hline
\end{tabular}

Fonte: Inserção do profissional farmacêutico na atenção básica de saúde: uma Revisão Integrativa, 2021.

Para organização e apreciação das variáveis aqui mapeadas esta pesquisa deteve de tabelas de análises, o que se fez executável a classificação de cunho metodológico dos trabalhos ora aqui alistados, cujo tema esteva direcionado para a inserção do profissional farmacêutico na saúde pública primária.

\section{Discussão}

A problemática aqui levantada proporcionou que houvesse o recrutamento de 12 trabalhos de títulos científicos, sendo os mesmos capazes de explicitarem informes relevantes que fossem capazes de solucionara a situação problema, inicialmente formulada, no presente estudo. As informações nesses achados foram eficientes, demonstrando direta e indiretamente a importância da inserção do profissional farmacêutico na atenção básica de saúde do Brasil. Fez-se perceptível ainda que, à 
atividade laborativa do profissional citado anteriormente é indispensável na atenção primária, ora que, corrobora para uma melhora na qualidade de vida dos usuários do SUS quando considerado a prática clínica.

Lopes, Staub \& Rossa (2018) esclarece que para o indivíduo desfrutar de um acesso na saúde pública de forma efetiva é necessário que haja uma sensibilização dos gestores e profissionais de saúde quando a inserção do farmacêutico, relatando ainda que na prática clínica a atuação de tal colaborador gera um impacto considerável e positivo não apenas no sucesso da farmacoterapia, bem como na humanização do cuidado através de medidas educacionais acessíveis.

Ainda análogo ao contexto da inserção farmacêutica, cabe mensurar que Pereira, Lima e Machado (2018), ao executarem uma pesquisa baseada no método histórico-comparado, desenvolvido a partir de três dimensões de análises: contesto estatal, trajetória e institucionalidade da descentralização e regionalização da saúde, bem como seus à condicionantes puderam determinar que no Brasil, percebe-se uma valorização da relação de cunho legal ou contratual na conformação das regiões de saúde, bem como na responsabilização dos gestores, sendo fundamental a acessibilidade do profissional farmacêutico nas áreas básicas de saúde.

Murakami \& Neto (2018), buscaram identificar e analisar as legislações e publicações científicas que relacionava-se a assistência farmacêutica no SUS tendo como profissional o farmacêutico como gestor desse processo, realçando seu papel na assistência farmacêutica desde sua legislação. A mesma contribuição supramencionada, pode determinar que a comunidade científica certifica que a inserção farmacêutica no SUS é considerada uma estratégia de vincular esses profissionais aqui compreendidos com os demais colaboradores da área da saúde, bem com os usuários dos serviços, assim como, sua atividade em medidas de intervenção na prevenção e promoção à saúde alusiva aos medicamentos.

Os dados aqui recrutados e as amostras devidamente apresentadas foram efetivamente capazes de demonstrar a relevância da inserção do farmacêutico na atenção primária, todavia existe certas necessidades em algumas gestões política. Caetano, Silva \& Luísa (2020) ao executarem um estudo baseado na análise temática de conteúdo e à luz do modelo ambiguidadeconflito de análise de implementação cujo objetivo fora analisar os níveis de ambiguidade e conflito em relação aos objetivos e meios necessários à implementação dos serviços farmacêuticos no âmbito da Atenção primária à saúde no município do Rio de Janeiro, puderam determinar que ainda se faz notório que haja esforços dos gestores em relação a consolidação da inserção farmacêutica. $\mathrm{O}$ mesmo estudo citado anteriormente frisa a relevância de um incremento no investimento em ações de cunho educativo permanente dos farmacêuticos e profissionais de apoio, que haja criação e institucionalização de diretrizes, metas e mecanismos para a inserção dos serviços farmacêuticos na atenção primária de saúde.

Fazendo um paralelo, cabe aqui destacar o estudo de Carvalho \& Leite (2017) que ao realizarem uma pesquisa transversal de abordagem quantitativa, com dados da pesquisa nacional sobre acesso, utilização e uso racional de medicamentes e serviços cujo objetivo fora caracterizar a força de trabalho da assistência farmacêutica na atenção básica do SUS determinaram que a profissionalização das atividades de gestão municipal na atenção básica é uma conquista na organização da força de trabalho da assistência farmacêutica. Porém, há uma relevante deficiência na composição da força de trabalho nas unidades responsáveis pela dispensação de medicamentos.

Diante da problemática explanada anteriormente e mediante análise das pesquisas aqui mensuradas, fomenta-se que é de suma importância que haja um engajamento ético da parte dos farmacêuticos para que essa acessibilidade seja efetiva. Molina, Hoffmann \& Finkler (2020) ao buscarem discutir e identificar os problemas éticos presentes no cotidiano dos farmacêuticos na atenção básica à saúde. Com isso foram capazes de explicitarem através de uma entrevista semiestruturada com 19 farmacêuticos, no município de Florianópolis, Santa Catarina que os problemas são alusivos à dimensão política do trabalho e da produção do cuidado, havendo envolvimento de questões pertinentes a estrutura do serviço, a processos de gestão e à maneira de organização da atenção à saúde. Ou seja, a análise ética comprometida com a resolução de problemas apontados, faz-se, relevante estratégia para que haja uma boa qualificação dos serviços farmacêuticos na atenção básica de saúde. 
Fazendo um paralelo ao examinar a amostragem aqui levantada e a prática da integralidade no cuidado do profissional aqui considerado, levando em consideração percebe-se que existe uma lacuna que deve ser sanada na atenção primária à saúde. Todavia, cabe mensurar o estudo de Neto \& Borges (2020) que ao analisarem os discursos de colaboradores farmacêuticos sobre a prática clínica-famacêutica nas Unidades Básicas de Saúde da prefeitura municipal de Vitória congruente ao princípio de integralidade puderam determinar que não existe uma uniformidade quando as práticas clínicas farmacêuticas. Diante disso, o estudo supramencionado fomenta a relevância da qualificação profissional, o direcionamento legal e a importância de se desfazer as limitações a título estrutural.

Foi possível ainda determinar que a avaliação farmacêutica de cunho básico ainda possui alguns desafios a serem analisados. Costa (2017), ao explanar uma síntese cuja temática estava voltada para a assistência farmacêutica básica, detectou que desafios são evidentes, sendo o acesso equitativo dos medicamentos, por exemplo, a estruturação dos serviços farmacêuticos, o melhoramento da logística e da gestão e o estabelecimento de ações voltadas ao cuidado farmacêutico nas unidades de saúde são proeminentes.

\section{Conclusão}

Diante do exposto, fomenta-se que ainda existe um aumento considerável de indivíduos realizando uso irracional de medicamentos e com à acessibilidade do profissional farmacêuticos na atenção primária de saúde esse cenário pode mudar significativamente. Visto que, sabe-se que o uso incorreto de medicamentos pode ser tornar um sério problema de saúde pública. Portanto, um dos fatores averiguados na presente pesquisa é que os profissionais da saúde citados acima alertam os usuários do SUS sobre os riscos do uso incorreto dos medicamentos intervindo sobre tal conduta alertando a sua gravidade e tentando adequar o plano terapêutico ofertado para um certo indivíduo.

A inserção farmacêutica tem influenciado muito sobre a atenção básica de saúde, fazendo-se necessário que as gestões de saúde pública prezem os serviços prestados por estes colaboradores da saúde humana, ora que assim minimize-se os usos incorretos de medicamentos prevenindo possíveis danos a saúde pública.

\section{Implicações práticas}

Sugere-se que realizem mais pesquisas cujo objetivo seja a complementação do presente estudo que apresentem informações alusivas a inserção profissional farmacêutica no âmbito da saúde pública básica da federação brasileira.

\section{Contribuição dos Autores}

YJM orientou e norteou a estratégia de pesquisa e recrutamentos dos achados juntamente com JSLC. JSLC realizou o recrutamento dos artigos frente a bibliografia reunida, advindos das bases de dados mencionadas nos métodos da presente revisão integrativa. A versão final do artigo científico foi aprovada por YJM. A escrita e todo conteúdo explicitado no presente estudo é de inteira responsabilidade dos autores.

\section{Declaração de conflito de interesses}

Os autores declaram a inexistência de conflito de interesses na elaboração da presente revisão integrativa.

\section{Agradecimentos}

Agradecemos primeiramente a Deus por sua supremacia e amor, aos nossos familiares e docente. 
Research, Society and Development, v. 10, n. 13, e155101320797, 2021

(CC BY 4.0) | ISSN 2525-3409 | DOI: http://dx.doi.org/10.33448/rsd-v10i13.20797

\section{Referências}

Caetano, M. C., Silva, R. M. D., \& Luiza, V. L. (2020). Serviços Farmacêuticos na Atenção Primária em Saúde à luz do modelo ambiguidade-conflito. Physis: Revista de Saúde Coletiva, 30, e300420.

Carvalho, M. N., Álvares, J., Costa, K. S., Guerra Junior, A. A., Acurcio, F. D. A., Costa, E. A., ... \& Leite, S. N. (2017). Força de trabalho na assistência farmacêutica da atenção básica do SUS, Brasil. Revista de Saúde Pública, 51, 16s.

Costa, K. S., Tavares, N. U. L., Nascimento Júnior, J. M. D., Mengue, S. S., Álvares, J., Guerra Junior, A. A., ... \& Soeiro, O. M. (2017). Avanços e desafios da assistência farmacêutica na atenção primária no Sistema Único de Saúde. Revista de Saúde Pública, 51, 3s.

Melo, D. O. D., \& Castro, L. L. C. D. (2017). A contribuição do farmacêutico para a promoção do acesso e uso racional de medicamentos essenciais no SUS. Ciência \& Saúde Coletiva, 22, 235-244.

Emiliano, J. P. M. (2013). Assistência farmacêutica e atenção farmacêutica: Novas perspectivas para o Farmacêutico. Revista de APS, 16(2).

de Matos Lopes, F. R., Staub, M. P., \& Roos, M. L. M. (2018, April). Atenção Farmacêutica e a Humanização da Assistência: A Influência do Profissional Farmacêutico na Acessibilidade à Informação. In I MOSTRA DE EXPERIÊNCIAS PROMOTORAS DE SAÚDE.

Maciel, A. P. P. (2018). Atuação cotidiana do farmacêutico na atenção primária à saúde Fortaleza.

Molina, L. R., Hoffmann, J. B., \& Finkler, M. (2020). Ética e assistência farmacêutica na atenção básica: desafios cotidianos. Revista Bioética, $28,365-375$.

de Freitas Nakata, K. C., \& da Silva, L. M. V. (2014). Avaliação da acessibilidade à assistência farmacêutica básica no município de Várzea Grande (Mato Grosso). Revista de Ciências Farmacêuticas Básica e Aplicada, 35(3).

de Barros Neto, S. G., \& Borges, L. H. (2020). A Prática da Integralidade no Cuidado Farmacêutico na Atenção Primária à Saúde. Ensaios e Ciência C Biológicas Agrárias e da Saúde, 24(1), 88-94.

Oliveira, N. D. P. (2014). Avaliação da assistência farmacêutica na atenção básica de saúde de Ceilândia-Distrito Federal.

Oliveira, L. C. F. D., Assis, M. M. A., \& Barboni, A. R. (2010). Assistência farmacêutica no Sistema Único de Saúde: da Política Nacional de Medicamentos à atenção básica à saúde. Ciência \& Saúde Coletiva, 15, 3561-3567.

Pereira, N. C. (2013). Monitoramento do desempenho dos serviços farmacêuticos na Atenção Primária à Saúde: buscando a qualificação da gestão (Doctoral dissertation).

Pereira, A. M. M., Lima, L. D. D., \& Machado, C. V. (2018). Descentralização e regionalização da política de saúde: abordagem histórico-comparada entre o Brasil e a Espanha. Ciência \& saúde coletiva, 23, 2239-2252.

Silva, D. Á. M., Mendonça, S. D. A. M., Oliveira, D. R. D., \& Chemello, C. (2018). A prática clínica do farmacêutico no núcleo de apoio à saúde a família. Trabalho, Educação e Saúde, 16, 659-682.

Souza, M. T. D., Silva, M. D. D., \& Carvalho, R. D. (2010). Revisão integrativa: o que é e como fazer. Einstein (São Paulo), 8, $102-106$.

Vieira, F. S. (2010). Assistência farmacêutica no sistema público de saúde no Brasil. Revista Panamericana de Salud Pública, 27, $149-156$. 